\section{EUS-B-Feinnadelaspiration ist zuverlässig und sicher}

Leong P et al. Endoscopic ultrasound findneedle aspiration by experienced pulmonogists: a cusum analysis. Eur Respir J 2017; 50: 1701102. doi:10.1183/13993003.01102-2017

Die transbronchiale, ultraschallgestützte Feinnadelaspiration (EUS TBNA) ist ein etabliertes Verfahren für die Abklärung intrathorakaler Auffälligkeiten. Konsensus-Leitlinien empfehlen die Kombination mit einem endoskopischen Zugang über den Ösophagus (EUS-B-FNA). In der Beobachtungsstudie mit einer $\mathrm{Cu}$ mus-Analyse kamen erfahrene Pulmonologen gut mit der Methode zurecht.

3 Fachärzte für Pulmonologie nahmen an der Studie teil. Sie verfügten über eine große Expertise mit der EUS TBNA und hatten jeder $\geq 150$ Eingriffe durchgeführt. Sie wiesen allerdings keinerlei Erfahrung mit der EUS-B-FNA auf und erhielten dafür kein gesondertes Training. Die Lernkurve der Ärzte bildete ein Cusum-Test grafisch ab. Eine akzeptable Performanz ist bei einer horizontalen und absteigenden Kurvenprogression gegeben. Studienendpunkte waren außerdem die Sensitivität und Spezifität der EUS-B-FNA für die Diagnostik von nodalen und nicht nodalen Läsionen (Definitionen nach Kemp).
137 Patienten wiesen 152 abzuklärende Läsionen auf. Unterschiedlichste Lungenareale und Lymphknotenstationen waren betroffen. Für die Diagnose „maligner Prozess“ ergaben sich 92 richtig positive, 40 richtig negative, 0 falsch positive und 5 falsch negative Ergebnisse. Bei einer hohen Malignom-Prävalenz von $70,8 \%$ resultierte eine Sensitivität von insgesamt $94,8 \%$ für die Diagnose eines malignen Prozesses. Unter den Zielregionen befanden sich 40 (27,6\%) außerhalb der Lymphknotenkarte der IASLC (International Association for the Study of Lung Cancer). Dabei handelte es sich überwiegend um intraparenchymatöse Massen. Für die Diagnose Malignom betrug die Sensitivität hier 92,9\%. Für IASCL-Lymphknoten lag die Sensitivität bei $89,9 \%$ und der negative prädiktive Wert bei $84 \%$. Die meisten Patienten erhielten die EUS-B-FNA zu diagnostischen Zwecken. Ob die EUS-B-FNA das Lymphknoten-Staging bei nicht kleinzelligen Bronchialkarzinomen verbessert, konnte daher nicht beurteilt werden.

Die Cusum-Kurven der Pulmonologen waren absteigend mit ähnlichen Verläufen. 2 Ärzte durchbrachen nach <20 Interventionen und 1 Arzt nach 30 Eingriffen die vordefinierte Horizontale als Marker für eine ausreichende Kompetenz. Nebenwirkungen kamen selten vor. Dabei handelte es sich um 1 Pneumothorax, 1 exazerbierte chronisch obstruktive Lungenerkrankung und 1 neu aufgetretenes Vorhofflimmern ohne hämodyna- mische Instabilität. Mediastinitiden und Ösophagusperforationen kamen nicht vor.

FAZIT

Mit der EBUS TBNA vertraute Ärzte lernten die EUS-B-FNA schnell und gewannen zuverlässig Gewebe für eine korrekte Diagnose. Die Methode war zudem komplikationsarm. Anwender sollten aber laut den Autoren die anatomischen Besonderheiten und das mögliche Komplikationsprofil studieren und sich von gastroenterologischen Kollegen beraten lassen. Validierte Assessments wie das EUSAT sowie die Einführung von Qualitätsstandards und strukturierten Trainingsprogrammen könnten zur Qualitätssicherung beitragen.

Dr. med. Susanne Krome, Melle 